\title{
Localize lesion of horner's syndrome based on anhydrotic pattern
}

\author{
Muhammad I. Basri, ${ }^{1 *}$ Emy Wardani, ${ }^{2}$ Hasmawaty Basir, ${ }^{2}$ Audry D. Wuysang ${ }^{2}$
}

\begin{abstract}
Objective: The current case series will discuss about two horner syndrome cases with a different pattern of anhydrotic.

Methods: Male 54 years old came with punctured and burned pain sensation at left hand for the last one year which radiated along the shoulder toward the tip of left fingers. There were $10 \mathrm{kgs}$ of weight loss. Neurological examination showed unequal pupil size $2.5 \mathrm{~mm} /$ $1.5 \mathrm{~mm}$, left ptosis, left hemifacial and left arm anhidrosis. Cervical MRI

Results: Case I MSCT Thorax showed suspicious of neurogenic tumor which resulted in CV T1 destruction and case II MSCT thorax showed left lung collapse due to pleural effusion

Conclusion: Anhidrotic pattern will be an essential factor in making a topical diagnosis for patients with Horner syndrome, which also can be a fundamental basis in choosing the next additional examinations required to find the etiology.
\end{abstract} showed burst fracture involved CV T1 pedicle.
${ }^{1}$ Department of Anatomy, Faculty of Medicine, Hasanuddin University, Makassar, Indonesia ${ }^{2}$ Department of Neurology, Faculty of Medicine, Hasanuddin University, Makassar, Indonesia

${ }^{*}$ Correspondence to:

ikbal_fkguh@yahoo.com

Received: 5 February 2019 Revised: 27 February 2019

Accepted: 10 March 2019

Available Online: 1 May 2019

Keywords: Anhidrotic pattern, Horner's syndrome, Sympathetic nerve

Cite this Article: Basri Ml, Wardani E, Basir H, Wuysang AD. 2019. Localize lesion of horner's syndrome based on anhydrotic pattern. Journal of Case Reports in Dental Medicine. 1(2): 36-39. D0l: 10.20956/jcrdm.v1i2.93

\section{Introduction}

Horner's syndrome is a disorder of the sympathetic nerves in the eyes and face, consisting of three symptoms: miosis, ptosis, and anhidrosis. This syndrome was first discovered in animals by French physiologist Claude Bernard in 1854. But Johann Friedrich Horner, an ophthalmologist, was the first to describe it completely as a parese oculosympathetic in 1869 . There are three types of Homer's syndrome: central, preganglionic, and postganglionic.

\section{Case Report}

\section{The first order neuron lesion (central type)}

The axons of the first order neurons begin in the posterolateral hypothalamus region leading to the lateral region of the brain stem and ending at the ciliospinal center of Budge and Waller in the intermediolateral substance of the C8-T1 spinal cord. The most frequent lesions in the hypothalamus are tumors and bleeding, which can cause Horner's syndrome with contralateral hemiparesis and contralateral hypesthesia. Lesions in the dorsal mesencephalon region (pontomesencephalic junction) cause Horner's syndrome accompanied by contralateral trochlear nerves. Wallenberg's syndrome (lateral medulla stroke) is the most frequent etiology of this type. Other symptoms that can be found are ipsilateral ataxia and contralateral hypalgesia. The most common spinal cord region lesions that cause Horner's syndrome are syringomyelia and spinal cord trauma. Other etiologies are myelitis, vascular malformations, demyelinating, tumor and infarction. ${ }^{1}$

\section{The second order neuron lesion (preganglionic type)}

The neuron of the second order neuron of the sympathetic nerve exits from the ciliospinal center of Budge and Waller C8-T1, and then through the pulmonary apex, rises through stellate ganglion, enters to the carotid sheath and synapse in the superior cervical ganglion which located at the bifurcation level of common carotid artery.

Pulmonary apex lesions that press the superior thoracic outlet provide symptoms of Horner syndrome with ipsilateral shoulder pain, medial paresthesias of the arms and fingers IV-V, and weakness or atrophy of the palm muscle. All of these signs and symptoms are known as Pancoast syndrome. The most common cause of Pancoast syndrome is a non-small cell lung carcinoma. Patients with the preganglionic syndrome and ipsilateral shoulder pain should be investigated for suspicion of neoplasm. ${ }^{1}$

\section{The third order neuron lesion (postganglionic type)}

The neuron axons of the third order of sympathetic nerves originate from the superior cervical ganglion, travel upward with the internal carotid artery, and then to the cavernous sinus. Furthermore, the axon goes along with the abducent 

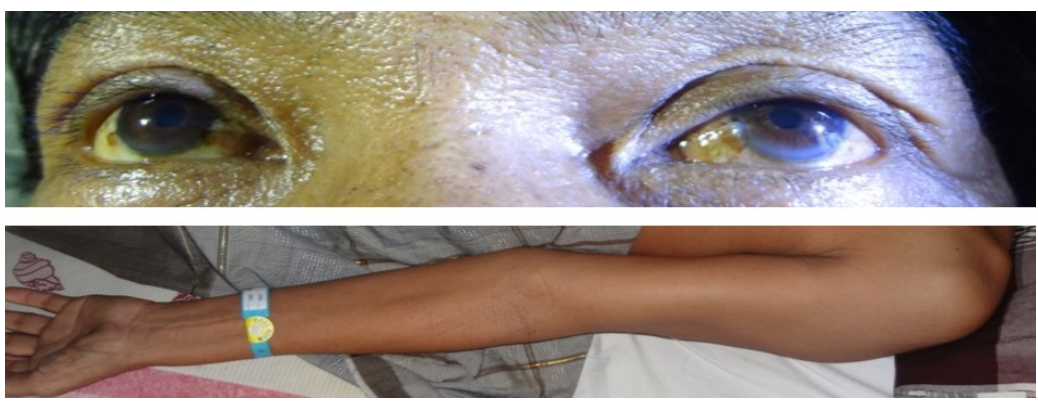

Figure 1 Case 1: The upper image shows the miosis of left pupil and left ptosis and the picture below was leftarm atrophy

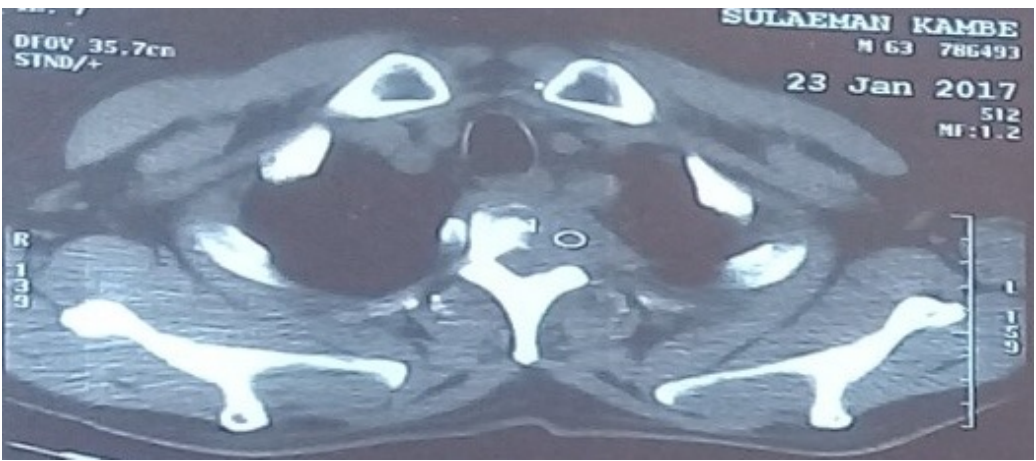

Figure 2 MSCT Thorax case 1: the appearance of isodense lesions $(33 \mathrm{HU})$ in the posterior mediastinum that produces CV T1. Impression was suspected neurogenic tumor

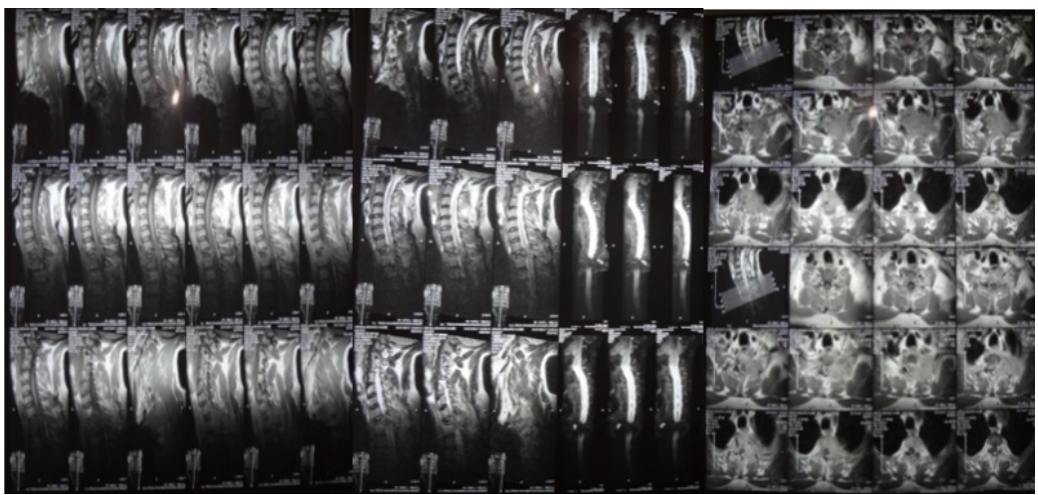

Figure 3 C-Spine MRI: Burst fracture involving CV T1 pedicle suspected metastasis, $\mathrm{C} 3-\mathrm{C} 4 \mathrm{CV}$ disc bulging that compresses the thecal sac, CV C4-C5 disc bulging that compress the thecal sac, MR-Myelography: CV-like spinal canal stenosis T1

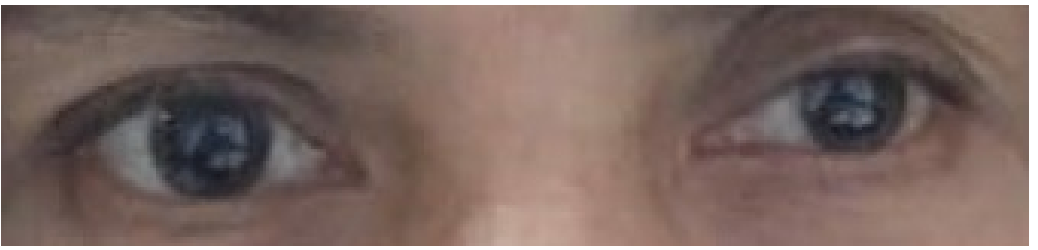

Figure 4 Showing left slight ptosis (case 2) and ophthalmic division of trigeminal nerve enters the orbital region with the nasociliary branch. The nasociliary nerve is divided into two long ciliary nerves travel laterally and medially, and then supply the iris dilator muscle. Horner's syndrome accompanied by ipsilateral facial pain or headache is known as the Raeder's paratrigeminal syndrome. ${ }^{2}$

Carotid dissection is a cause which has to be considered if Horner's syndrome is accompanied by ipsilateral facial or head pain, carotidynia, and dysgeusia. Carotid thrombosis that interferes with blood flow to the superior cervical ganglion or carotid plexus causes ipsilateral oculosympathetic weakness with or without pain. Other causes include cluster headache, small-vessel ischemia, neuropathy, intraoral trauma, cavernous sinus lesions, superior orbital fissures and apex orbital.

\section{Clinical Manifestation}

Ptosis. The weakness of Muller's muscle causes upper palpebral drooping with a moderate degree. The lower palpebra is slightly raised, so-called "upside-down ptosis" or “reverse ptosis". Horner's syndrome does not cause visual impairment.

Miosis. Disorders of sympathetic input to the iris dilator muscles cause ipsilateral miosis. Anisocoria will be more visible if it is dark or also called dilation lag. Normal pupils will be rapidly dilated whereas sympathetically denervated pupils will experience a delay of dilation.

Anhidrosis. Changes in vasomotor and sudomotor characteristics of facial skin and or ipsilateral arms. Sympathetic denervation increase skin temperature due to loss of vasomotor control. Furthermore, blood vessel dilatation occurs. Others symptom such as facial flushing, conjunctival hyperemia, and epiphora may be found. Occasionally, ipsilateral facial and neck skin has a low temperature and appears paler than the normal side. This is caused by the supersensitivity of the denervated blood vessels to adrenergic which causing vasoconstriction. ${ }^{1}$

\section{Diagnosis}

Pharmacologic tests can help establish a diagnosis and determine the location of lesions in Horner's syndrome: cocaine blocks the reuptake norepinephrine released by the sympathetic nerve. Norepinephrine accumulates at the receptor, causing normal pupillary dilation, apraclonidine, as a weak alpha- 1 adrenergic agonist. So that there is a minimal constriction of normal pupils and dilation of Horner's pupils (supersensitivity to the iris dilator muscle), hydroxyamphetamine, to distinguish post-ganglion type to others. 


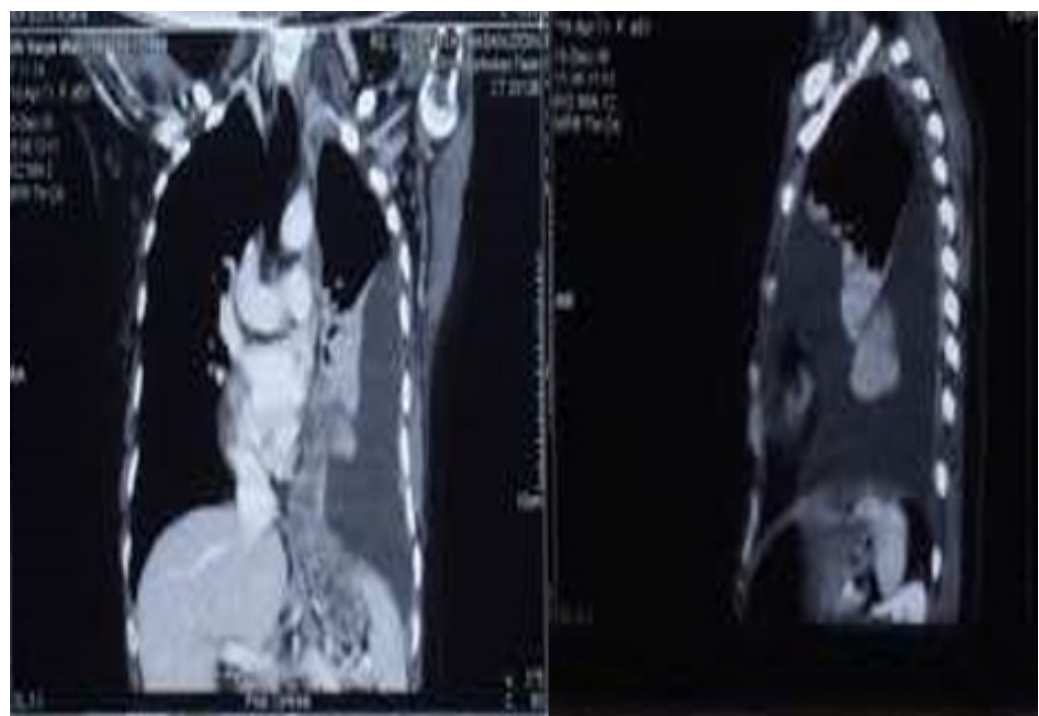

Figure 5 Thoracic MSCT in case 2

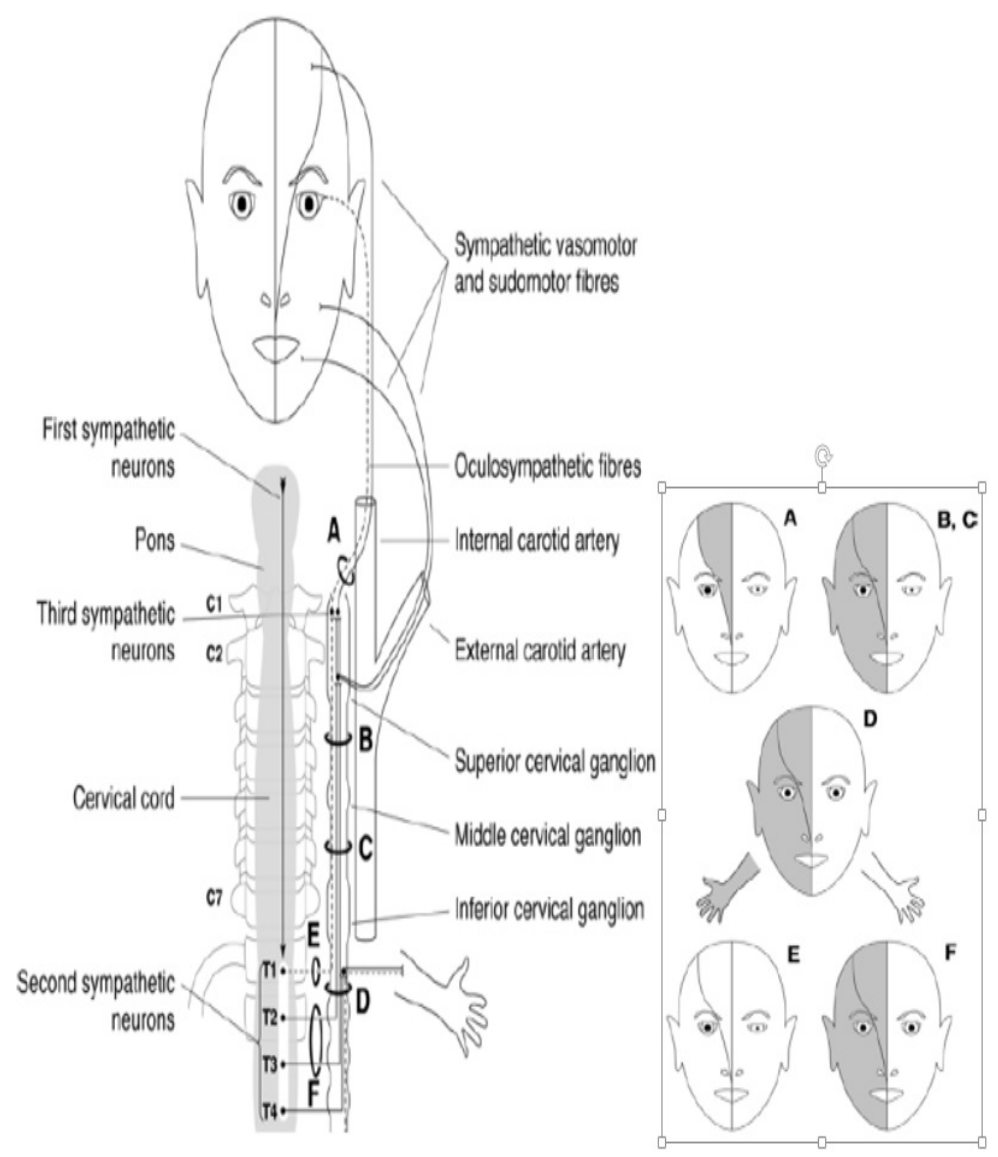

Figure 6 Sympathetic pathways and anhidrosis pattern in Horner's syndrome
Hydroxyamphetamine causes norepinephrine secretion from the postganglionic adrenergic nerve, causing mydriasis in normal pupils. If there is disruption in post-ganglion terminal nerve endings, it can cause no expenditure of norepinephrine. Whereas in central and preganglionic type, the nerves are still intact. ${ }^{1-3}$

\section{Case 1}

Mr. KS, 54 years old, underwent pain on the left arm since a year before admission to the hospital (NPRS 9). Pain radiates from the shoulder to the fingertips, especially the 4th and 5th fingers, punctured and hot sensation. It was felt throughout the day, burdensome if active and decreases when lying down and taking medication (NPRS 5). Ten kilograms less weight in the last 3 months. A history of prolonged coughing, diabetes, heart disease, and trauma was denied. Internal status within normal limits. Neurological examination was normal 2.5 $\mathrm{mm} / 1.5 \mathrm{~mm}$ anisocoria pupil $\mathrm{RCL}+/+$ normal; Normal RCTL $+/+$, the result of funduscopy was papillary ODS edema (-); VODS 6/6, Left ptosis; Motoric strength decrease on the left finger flexion decreases (2-3); sensibility was hemihypesthesia dermatome region C8-T1; Autonomous function was left hemifacial anhydrosis and left superior extremity. There was left-arm atrophy (brachial, antebrachial and palmar region).

WBC laboratory examination: 16,3,03 / mm3; RBC: 4.75 xl06 / ul; HGB: 14.2 g / dl; PLT: 346 xl03 / ul. Chest radiograph was dilatation et elongation aortae, heart and lung were normal. Bronchoscopic results were normal bronchi and its branches, blunt secondary carina. EMNG results were severe axonal neuropathy multiplex, compression of left radix C8Th1. Bronchus Cycle Culture Results were Grampositive (positive $1+$ ) and gram-negative (positive $1+), P M N>25$ lpk, epithelial cells $<10$ lpk, smear staining (-), no growth of anaerobic bacteria. Cytology of Bronchial Fluids was inflammatory lesions. So the diagnosis was Horner's Syndrome due to mediastinum tumor and compress the sympathetic truncus.

The management of this patient were celecoxib $200 \mathrm{mg} / 12$ hours p.o, gabapentin $300 \mathrm{mg} / 24$ hours p.o., amitriptyline $12.5 \mathrm{mg} / 12$ hours p.o, omeprazole $40 \mathrm{mg} / 12$ hours / intravenously, mecobalamin 1 ampule / 24 hours / intravenously.

\section{Case 2}

Mrs. SK, 46 years old, underwent an intermittent pain on the left arm since 1 year ago, feeling the pierced sensation. She felt dyspnoea a month ago. 
The internal status was within normal limits. Neurological examinations were $3 \mathrm{~mm} / 2 \mathrm{~mm}$ anisocoria round pupils; Normal light reflexes, funduscopy was no papillary ODS edema; VODS was $6 / 6$, left ptosis; the motoric functions was the strength of the left finger flexion decreases (2-3); sensibility was hemihypesthesia dermatome region C8-T1; Autonomous function was left hemifacial anhydrosis. There was left-arm atrophy (brachial, antebrachial and palmar region).

The result of the laboratory test was within normal limits. Chest radiograph was bilateral bronchopneumonia and pleural effusion. The impression of thoracic MSCT was a pulmonary collapse, and pleural effusion. Compensate right lung emphysema. There were bulging disc posteriorly at CV level C2-C3, C3-C4, and C4-C5 which compresses the thecal sac and spinal cord and irritates both nerve roots in the C-Spine MRI. Signs of the posterior bulging disc on CV C5-C6 which suppress the thecal sac and spinal cord. Facet joint edema at CV level C4-C5 left and C6-C7. There was a cervical spondylosis. So, the diagnosis was Horner's Syndrome due to left lung collapse. It compressed preganglion of the sympathetic nerve. The management of this patient was meloxicam 15 mg / 24 hours p.o., gabapentin 300 mg / 24 hours p.o., mecobalamin 1 tablet / 24 hours p.o., She was consulted to a pulmonologist.

\section{Discussion}

Based on the results of the history, physical examination and investigation. Both of the patients showed complete symptoms of Horner's syndrome and brachialgia. Horner's syndrome, accompanied by brachialgia, was a typical sign of second-order sympathetic nerve lesions (preganglionic type). The different patterns of anhidrosis in the two patients showed the different location of the lesion. ${ }^{4}$

Vasomotor and sudomotor fibers that supply the face come out at the T2-T3 level, while the preganglion oculosympathetic neurons originate from $\mathrm{T}-1$. The pre-ganglion fibers that supply the upper limb originate from T-4 downwards. They synapse in the stellate ganglion located more caudally (at level C7).

Based on these anatomical considerations, the location of the lesion along the sympathetic pathway can be detected based on the patient's clinical symptoms. In case 1 , the patient's anhydrosis pattern was in the ipsilateral face and right arm. The estimated lesion level is as high as D. But in case 1 , there are ipsilateral miosis pupils. So the possibility of lesions also covers region E. From the results of the MRI C-Spine examination, there is a burst fracture involving the CV T1 pedicle, which is thought to suppress the pre-ganglion oculosympathetic neurons (ipsilateral miosis). Posterior mediastinal tumors which result in an emphasis on the sympathetic pathway as high as the $\mathrm{D}$ region.

In case 2, left hemifacial anhidrosis showed preganglionic lesions between the stellate ganglion and the superior cervical ganglion (region $\mathrm{B}$ or $\mathrm{C}$ ). In the sympathetic trunk consists of the oculosympathetic pre-ganglion neurons and the ipsilateral facial vasomotor and sudomotor fibers. This is in accordance with the patient's clinical symptoms, namely ipsilateral miosis and ipsilateral facial anhidrosis. Atelectasis in the left lung due to massive pleural effusion causes compression of the sympathetic nerve that passes through the pulmonary apex. So the possibility of suppression of sympathetic nerves before region $\mathrm{C}$.

\section{Conclusion}

The anhidrosis pattern of Horner's syndrome patients is very important in determining the topical diagnosis. It can help us to choose the right examination to find the etiology.

\section{Aknowledgmet}

None.

\section{Conflict of Interest}

The authors report no conflict of interest.

\section{References}

1. Kanagalingam S, Miller NR. Horner syndrome: clinical perspectives. Dove Press J Eye \& Brain 2015: 35-46.

2. Liu GT, Volpe NJ, Galetta SL. Neuro-ophthalmology: diagnosis and management. Saunders Elsevier; 2010. p. 428-441.

3. Schiefer U, Wilhelm H, Hart W. Clinical neuroophthalmology a practical guide: diagnosis of pupillary disorders. Springer; 2007. p. 60-64.

4. Fringeli Y, Humm AM, Ansorge A, et al. Harlequin sign concomitant with horner syndrome after anterior cervical discectomy: a case of intrusion into cervical syympathetic system. J Neurosurg Spine 2017;26: 684-687. 